\title{
Gravity, Time, Mass and Super Force United
}

\section{Paul TE Cusack*}

Dule, 1641 Sandy Point Rd, Saint John, NB, E2K 5E8, Canada

\section{Abstract}

The Superforce has long been the "The Holy Grail" of Physics. Here is more on its solution. Using AT Math, this paper shows the mathematical derivation of the Superforce as being the area bound by the golden mean parabola and the logarithmic functions from 1 to $\mathrm{Pi}$. Using calculus on the Clairnaut equation, and our knowledge of the equation for the gravitational constant, Using the equation derived in Astrotheology Cusack's Universe, E=1/t, Time and gravity are shown to be finally united.

Keywords: Clairnaut; Time; Golden mean; Reynold's gravity; Centroid

\section{Introduction}

In Astrotheogly, Cusack's universe, we proposed that the Superforce, the force that unites the four fundamental forces in the universe, is $\mathrm{F}=8 / 3$. In this brief paper, we continue with those calculations using simple calculus to show that the superforce is the force times the energy bound by the Golden Mean Parabola and the logarithmic functions. The centroid of this area is the time vector, which provides a proof for the simple, important equation, $\mathrm{E}=1 / \mathrm{t}$. We begin with the Clairnaut equation.

\section{Cusack-Clairnaut Differential Equation}

\section{$\mathrm{d}^{2} \mathrm{E} / \mathrm{dt}^{2}-\mathrm{E}=0$}

$\mathrm{G}-\mathrm{E}=0$

Gravity is the acceleration of energy, or the second derivative of displacement. What then is the Velocity of energy, or the first derivative of the displacement?

We know from AT Mathematics [1]:

$y=y^{\prime}=y^{\prime \prime}$

Integrating:

$\int \mathrm{d}^{2} \mathrm{E} / \mathrm{dt}^{2}=\int \mathrm{E}$

$\mathrm{G}^{2} / 2=\mathrm{E}^{2} / 2$

$\mathrm{G}=\mathrm{E}$

And,

$\mathrm{dE} / \mathrm{dt}=\mathrm{G}^{2} / 2$

$\mathrm{G}=\pi / \operatorname{Ln} 1.618$

$=6.52$

So,

$6.52^{2} / 2=4.263 \sim \mathrm{cuz}$

Time

$\int \mathrm{dE}^{2} / \mathrm{dt}^{2}=\int \mathrm{E}$

$\mathrm{dE} / \mathrm{dt}=\mathrm{E}^{2} / 2$

But $\mathrm{E}=1 / \mathrm{t}$

$\mathrm{E}=\mathrm{t}=1$
From the Golden Mean Parabola:

$\mathrm{dE} / \mathrm{dt}=1^{2} / 2=\mathrm{t}$ at $\mathrm{E}_{\min }=1.25$

The Velocity of energy is time $t$.

This evokes the Golden Mean Parabola minimum. Plugging the Gravitational constant (less electromagnetic forces) into the golden mean parabola, we have:

$\mathrm{t}^{2}-\mathrm{t}^{2}-1=\left(6.52^{2} / 2\right)-(6.52 / 2-1=4.486=$ Mass M.

Using Einstein's Equation, we can derive the proportional amount of mass in the universe.

$\mathrm{E}=\mathrm{Mc}^{2}$

$=4.486 \times 2.9979^{2}$

$=403=$ Reynold's Number

This is the energy necessary to get the Ether to flow.

Now, to unite gravity and time.

We now know,

$\mathrm{G}=\pi / \operatorname{Ln} 1.618$

And,

$\mathrm{t}=\pi$ and $\mathrm{t}=1.618$ and $\mathrm{t}=1$ Refer to Figure 1 .

$\int_{(1-\pi)} \operatorname{Ln} \mathrm{t}-\int_{(1.618-\pi)} \mathrm{t}^{2}-\mathrm{t}-1=\mathrm{E} \times \mathrm{t}$

$\left[-1 / 2 \mathrm{t}^{2}\right]-\left[2 \mathrm{t}^{3} / 2-\mathrm{t}^{2} / 2-1\right]=\mathrm{E} \times \mathrm{t}$

$\mathrm{E}=1 / \mathrm{t} \mathrm{E}=\mathrm{t}=1$

Let $\mathrm{t}=1$

$=8 / 3=\mathrm{F}=\sin \mathrm{t}=\sin 1=$ Superforce

Since $\mathrm{E}=\mathrm{t}$

*Corresponding author: Cusack PTE, Independent Researcher, BSc E, DULE, 1641 Sandy Point Rd, Saint John, NB, Canada E2K 5E8, Canada, Tel: (506) 2143313; E-mail: st-michael@hotmail.com

Received March 31, 2017; Accepted November 28, 2017; Published December 04, 2017

Citation: Cusack PTE (2017) Gravity, Time, Mass and Super Force United. Fluid Mech Open Acc 4: 181. doi: 10.4172/2476-2296.1000181

Copyright: @ 2017 Cusack PTE. This is an open-access article distributed under the terms of the Creative Commons Attribution License, which permits unrestricted use, distribution, and reproduction in any medium, provided the original author and source are credited. 


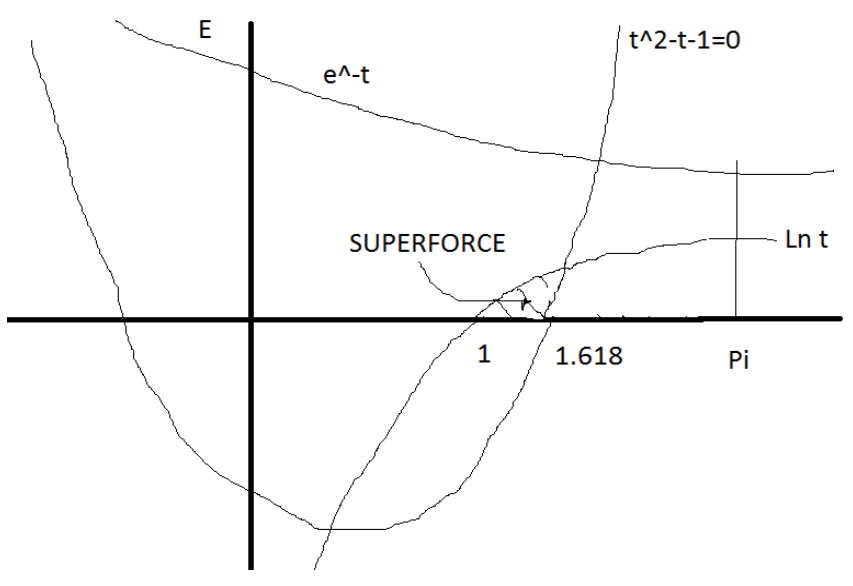

Figure 1: Superforce: Areas between In and golden mean curves.

Centroids of Area
$y=(3 / 2) h$
$=3 / 8(0.618)$
$=0.927$
$x=0.4 y$
$=(0.4)(0.927)=0.37$
Time-Energy Vectror=
SqRt[0.927^2-).37^2]
$=0.998^{\sim} 1$
$F=E x t=1$
$E=1 / t$

Figure 2: Centroid of superforce area.

From the solution of the Navier-Stoke's Fluid Mechanics problem solved by the author in a previous paper:

$\mathrm{F}=\mathrm{E} \times \mathrm{t}=\sqrt{2} 2.666=1.633$ Solution to the Navier-Stokes Problem [2]

Now we compute the centroid of the Superforce area under the curves (Figure 2).

\section{Eta Prime Mesons}

We know from Matrix Theory of Structural analysis,

$\mathrm{dU}=\delta \mathrm{W}-\int \rho \delta \mathrm{u} \mathrm{u}^{\prime \prime} \mathrm{dv}$

Strain energy $=$ Work - Dynamic load

$\varepsilon \alpha\left[\mathrm{F}_{\text {static }}-\mathrm{F}_{\text {dynamic }}\right]$

$[\Delta \mathrm{L} / \mathrm{L}]=[\mathrm{Fs}]-\int \rho \delta \mathrm{u} \mathrm{u} \mathrm{u}^{\prime \prime} \mathrm{dv}$

$$
\mathrm{L}=1 / 2
$$

$\mathrm{F}_{\text {static }}=\mathrm{ks}=0.4233$

$$
\mathrm{F}_{\text {dynamic }}=\sin \theta=2.668=8 / 3
$$

So,

$\int \rho \delta \mathrm{u} \mathrm{u}^{\prime \prime} \mathrm{dV}=\mathrm{F}_{\text {dymanic }}=\mathrm{Ma}=\sin \theta$

$\mathrm{Ma}=\int \rho \delta \mathrm{u} \mathrm{u}^{\prime \prime} \mathrm{dv}$

Take the derivative:
$M a^{\prime}=\rho \delta u u^{\prime \prime} d v$

Since we know $\mathrm{y}=\mathrm{y}^{\prime}=\mathrm{y}^{\prime \prime}$ etc.

$\mathrm{Mv}=\rho \mathrm{s}$ a dv

$1=\rho / M^{\star} s^{\star} a / v d v$

$1=$ Vol. $(\mathrm{s})(0.8415 / 0.8415) \mathrm{dv}$

$1=\mathrm{s}^{3} \mathrm{~s} \mathrm{dv}$

$1 / \mathrm{dv}=\mathrm{s}^{4}$

$1 / \mathrm{a}=\mathrm{s}^{4}$

$1 / 0.8415=\mathrm{s}^{4}$

$s=957.8=\eta{ }^{\prime}=$ Eta Prime Meson $\mathrm{MeV}$

So,

$\mathrm{F}_{\text {dynamic }}=\mathrm{M} \mathrm{p}^{+}+\Delta \mathrm{E}$

$=957.8 \mathrm{MeV}+0.1980$

$\mathrm{E}+\Delta \mathrm{E}=\eta^{\prime}+\tau$

Hamilton's K.E $=1 / 2 \int \rho \mathrm{v} \times \mathrm{v} d \mathrm{v}$

K.E. $=1 / 2 \mathrm{Mv}^{2}=1 / 2 \int \rho \mathrm{s}^{2} \mathrm{dv}$

$\mathrm{Mv}^{2}=\int \rho \mathrm{s}^{2}$

$\mathrm{Mv}^{2}=\mathrm{M} / \mathrm{s}^{3} \times \mathrm{s}^{2}$

$\mathrm{v}^{2}=1 / \mathrm{s}$

$v^{2} s=1$

$\mathrm{s}=1 / \mathrm{v}^{2}=1 / 0.707=\sqrt{ } 2$

$\varepsilon_{\mathrm{x}}=\varepsilon_{\mathrm{y}}=1$

$\mathrm{s}=\varepsilon_{0}{ }^{2}$

Now,

K.E. $=1 / 2 \int \rho \mathrm{v} \times \mathrm{v} d \mathrm{v}$

$1 / 2 \mathrm{Mv}^{2}=1 / 2(1.27) \mathrm{v} \mathrm{s}^{2}$

$1 / 2(4.486)(0.8415)=1.27 / 2 \times \mathrm{s}^{2}$

$\mathrm{s}=1.8794$

$\mathrm{s}=\Delta \mathrm{L}$

$\Delta \mathrm{L} / \mathrm{L}=\varepsilon_{0}=\mathrm{s}$

$\Delta \mathrm{L} /(1 / 2)=1.8794$

$\Delta \mathrm{L}=119.6=$ falilure stress

$\sqrt{ }\left[(0.8466)^{2}+(0.8466)^{2}\right]=119.7>119.6$.

Mass of Largest Element in Periodic Table of the elements.

The Superforce, Hamilton's Kinetic energy, is stored in elementary particles. This is computational evidence for the Superforce $[3,4]$.

\section{Conclusion}

So we see that the Superforce is simply the area bound by the two important equations of the golden mean parabola and the natural logarithm equations which fully describe the mathematical universe. The energy and time vectors are equal to 1 . This paper unites gravity, 
time and the Superforce. K.E. developed by the Superfoce is stored in elementary particle mass.

\section{References}

1. Cusack PTE (2016) Astrotheology, Cusack's Universe. J Phys Math.
2. Cusack PTE (2016) The Navier-Stoke's Clay Institute Problem Solution. J Phys Math.

3. Przemieniecki JS (1962) Theory of matrix Structural Analyisis. Dover NY.

4. Ludwig W, Falter C (1995) Symmetries in Physics, Group Theory Applied to Physics Problmes, Springer. 